\title{
Nonlinear Tracking Control for a Hard Disk Drive Dual-Stage Actuator System
}

\author{
Jinchuan Zheng, Minyue Fu, Fellow, IEEE, Youyi Wang, Senior Member, IEEE, \\ and Chunling Du, Senior Member, IEEE
}

\begin{abstract}
This paper presents a nonlinear tracking control method for a hard disk drive dual-stage actuator (DSA) system that consists of a voice coil motor (VCM) actuator and a piezoelectric (PZT) microactuator. Conventional track seeking controllers for DSA systems were generally designed to enable the VCM actuator to approach the target track without overshoot. However, we observe that this strategy is unable to achieve the minimal settling time when the target tracks are beyond the PZT actuator stroke limit. To further reduce the settling time, we design the VCM actuator controller to yield a closed-loop system with a small damping ratio for a fast rise time and certain allowable overshoot. Then, a composite nonlinear control law is designed for the PZT actuator to reduce the overshoot caused by the VCM actuator as the system output approaches the target track. Experimental results show that the proposed dual-stage servo outperforms the conventional dual-stage servo in short-span seeking and, additionally, achieves better track following accuracy than the VCM only single-stage servo.
\end{abstract}

Index Terms-Dual-stage actuator (DSA), hard disk drive (HDD), motion control, nonlinear control, saturation.

\section{INTRODUCTION}

$\mathbf{T}$ HE CONTINUOUS increase of track density and highspeed data access in hard disk drives (HDDs) requires the head position accurately maintained along the track center (track following) and swiftly moved from one track to another (track seeking). However, the conventional voice coil motor (VCM) only single-stage servo makes it hard to provide high performance due to its mechanical resonance modes, various disturbances, and noise in HDDs. The dual-stage actuators (DSAs) are introduced to overcome the limitation [1], [2]. In DSA servo systems, the VCM actuator is used as the primary stage to provide long track seeking but with poor accuracy and slow response time while the secondary stage such as a piezoelectric (PZT) microactuator [3] is used to provide higher precision and faster response but with a stroke limit. By combining the DSA system with properly designed controllers, the two actuators are complementary to each other and the defects of one actuator can be

Manuscript received January 18, 2007, revised January 22, 2008. Current version published October 8, 2008. Recommended by Technical Editor M. Tomizuka.

J. Zheng and M. Fu are with the School of Electrical Engineering and Computer Science, The University of Newcastle, Callaghan, NSW 2308, Australia (e-mail: jinchuan.zheng@newcastle.edu.au; minyue.fu@newcastle.edu.au).

Y. Wang is with the School of Electrical and Electronic Engineering, Nanyang Technological University, Singapore 639798, Singapore (e-mail: eyywang@ntu.edu.sg).

C. Du is with the Agency for Science, Technology and Research (A*STAR) Data Storage Institute (DSI), Singapore 117608, Singapore (e-mail: du_chunling@dsi.a-star.edu.sg).

Color versions of one or more of the figures in this paper are available online at http://ieeexplore.ieee.org.

Digital Object Identifier 10.1109/TMECH.2008.919823

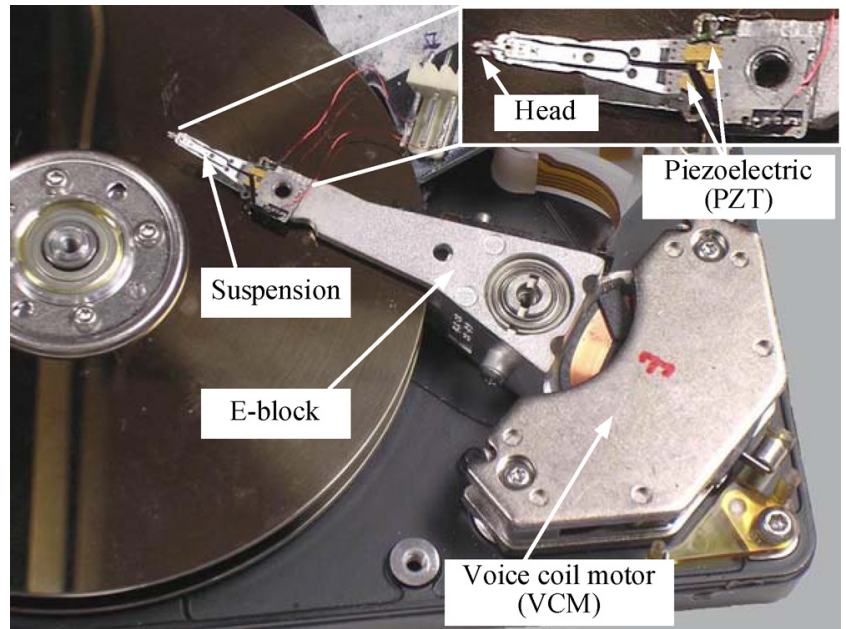

Fig. 1. HDD DSA with a PZT microactuator (throughout this paper, we will refer to the combined VCM and E-block assembly as VCM actuator and the combined PZT and suspension assembly as PZT actuator).

compensated by the merits of the other one. Therefore, the DSA system can provide both large displacement, high positioning accuracy, and fast track seeking.

It is a challenging task to design the DSA controllers to yield an optimal performance because of the specific characteristics: 1) The DSA system is a dual-input single-output (DISO) system, which means that, for a given desired trajectory, alternative inputs to the two actuators are not unique. Thus, a proper control strategy is required for control allocation. 2) The secondary actuator typically has a very limited travel range, which results in the actuator saturation problem. A variety of approaches have been reported to deal with the DSA control problems. For example, control design for track following and settling can be found in [4]-[6]. The secondary actuator saturation problem was explicitly taken into account during the control design [7], [8]. In [9], a decoupled track seeking controller using a three-step design approach is developed to enable high-speed one-track seeking and short-span track seeking for a dual-stage servo system. The control design for the PZT actuator by minimizing the destructive interference is proposed in [10] to attain desired time and frequency responses.

In this paper, we study a dual-stage HDD with a push-pull PZT microactuator as shown in Fig. 1. It consists of a VCM actuator as the primary stage and a PZT actuator as the secondary stage. The PZT is located between the suspension and the Eblock, which is moved by the VCM. The two actuators are, respectively, driven through a PZT amplifier and a VCM driver. The VCM driver has a voltage input limit of $\pm 3.5 \mathrm{~V}$. The PZT 

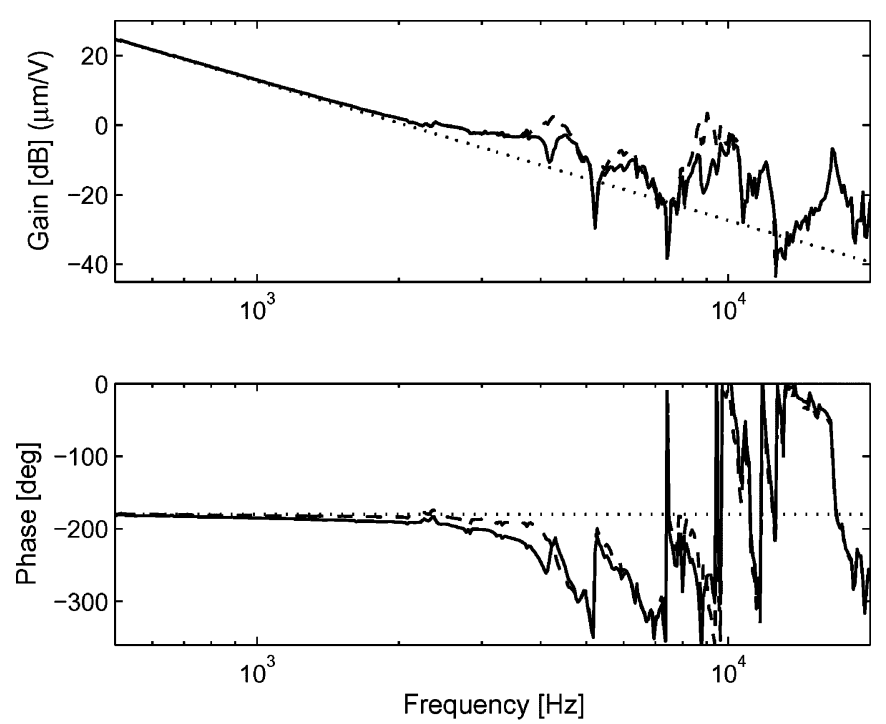

(a)
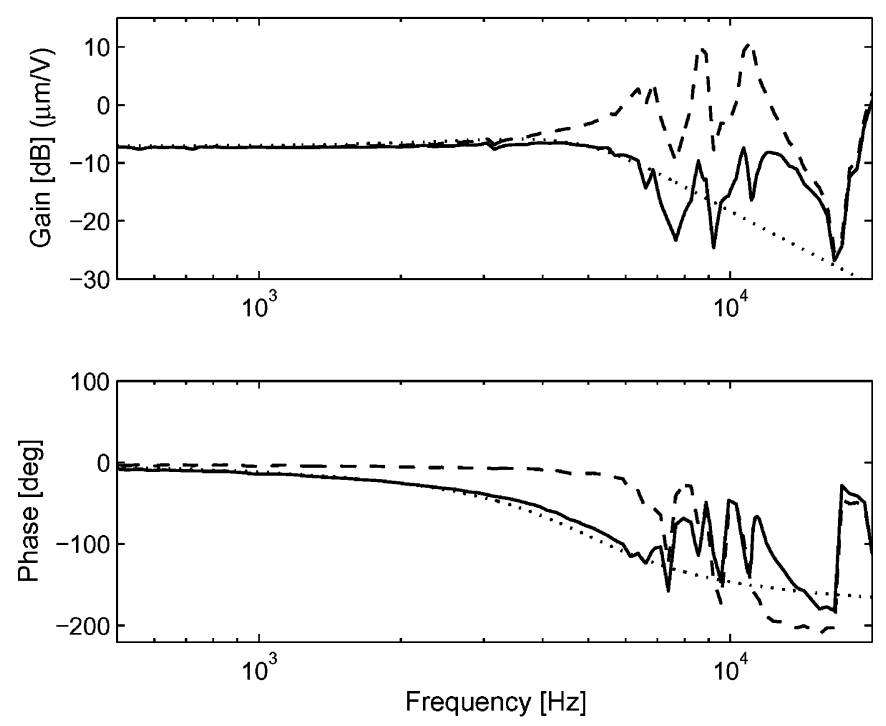

(b)

Fig. 2. Frequency responses of a HDD DSA system (dashed line: measured response; solid line: measured response with resonance compensator; dotted line: simulated response with resonance compensator). (a) VCM actuator. (b) PZT actuator.

actuator has a stroke limit of $\pm 0.5 \mu \mathrm{m}$ and the PZT amplifier has a voltage input limit of $\pm 1.5 \mathrm{~V}$. The head position is measured using a laser Doppler vibrometer (LDV) in our experiment setup. We assume that the coupling effects between the two actuators are negligible, and then, measure the frequency responses of the VCM and PZT actuator separately, which are shown with the dashed lines in Fig. 2.

By far, most of the work on the DSA track seeking control to follow a step command input is based on the strategy that the VCM actuator control loop is designed to have little overshoot, and the PZT actuator control loop is designed to follow the position error of the VCM actuator [9]-[11]. Under this conventional strategy, the total settling time can be reduced by the time that it takes for the PZT actuator to reach its stroke limit. However, we

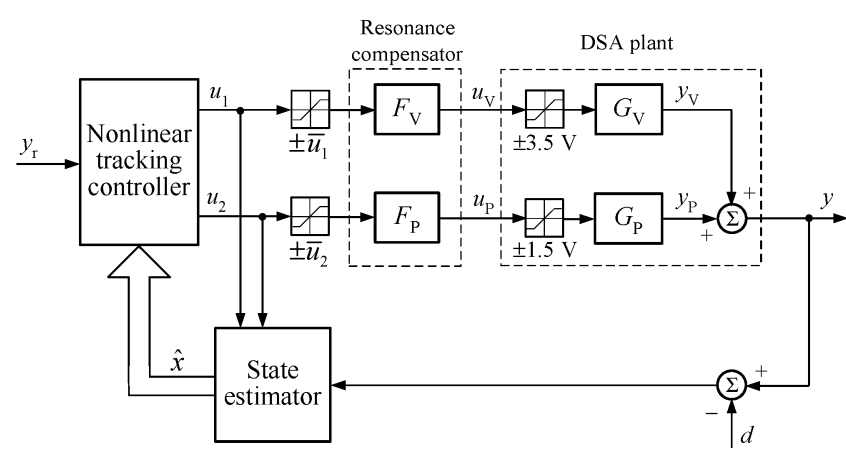

Fig. 3 Block diagram of a complete DSA control system.

observe that when the target track is beyond the PZT actuator travel range, this strategy is unable to minimize the total settling time because the PZT actuator can make little contributions due to its very limited travel range. To further reduce the settling time under this circumstance, we propose that the VCM actuator controller can be designed to yield a closed-loop system with a small damping ratio for a fast rise time allowing a certain level of overshoot, and then, as the VCM actuator approaches the target track, the PZT actuator control loop is used to reduce the overshoot caused by the VCM actuator. In this way, the total settling time is much less than that of the conventional control provided that the overshoot is within the PZT actuator stroke limit.

To perform the aforementioned control strategy, we will use the DSA control structure in Fig. 3, which consists of resonance compensators, a state estimator and a nonlinear tracking controller. The design of these control blocks will be presented in Section II. Section III shows the experimental results. Conclusions are given in Section IV.

\section{Nonlinear Tracking CONTROl Design}

Our objective here is to design the controllers in Fig. 3 such that the two actuators cooperate to enable head position $y$ to track a step command input of amplitude $y_{r}$ rapidly without exhibiting a large overshoot. In this section, we first describe the resonance compensation in Section II-A and state estimator in Section II-B for the DSA system. Then, Section II-C presents the proximate time-optimal control law to yield a VCM actuator closed-loop system with a small damping ratio so as to achieve a quick rise time. Finally, we develop a composite nonlinear control law with a step-by-step design procedure in Section II$\mathrm{D}$ and $\mathrm{E}$ for the PZT actuator, which can reduce the overshoot caused by the VCM actuator as the head position approaches the target track.

\section{A. Resonance Compensation}

The resonances of the E-block and the suspension exert adverse effects on the tracking performance. Here, we use a compensator with cascaded notch filters to actively damp the resonances. The transfer function of the resonance compensator is 
given by

$$
F_{V, P}=\prod_{i=1}^{n} \frac{s^{2}+4 \pi \zeta_{1 i} f_{i} s+\left(2 \pi f_{i}\right)^{2}}{s^{2}+4 \pi \zeta_{2 i} f_{i} s+\left(2 \pi f_{i}\right)^{2}}, \quad \zeta_{1 i}<\zeta_{2 i}
$$

where $f_{i}$ (in hertz) denotes the resonance frequency, and $\zeta_{1 i}$ and $\zeta_{2 i}$ are the damping ratios chosen to notch the resonance peak. We have implemented an $F_{V}$ to moderately dampen the main resonance modes at $4.2,6$, and $9 \mathrm{kHz}$ in the $\mathrm{VCM}$ actuator such that the compensated VCM model involves no extra phase lag within $1 \mathrm{kHz}$ while the compensated modes cause insignificant vibrations. Similarly, an $F_{P}$ is implemented to largely dampen the resonance modes at $6.8,8.8$, and $11.1 \mathrm{kHz}$ in the PZT actuator. The solid lines in Fig. 2 show the measured frequency responses of the VCM and PZT actuator after resonance compensation. From the measured response with resonance compensation, we observe that the compensated VCM actuator and PZT actuator can be reliably approximated as a pure double integrator and a second-order model, respectively, in the frequency range of interest. Furthermore, similar to [11], we place two saturation blocks before the resonance compensators and set the saturation levels $\bar{u}_{1}$ and $\bar{u}_{2}$ sufficiently small such that the control signals $u_{V}$ and $u_{P}$ never exceed the voltage limits of the VCM driver and PZT amplifier, respectively. In this way, we can treat the DSA with resonance compensation as a linear DISO system, which is represented in a state-space form as

$$
\left\{\begin{aligned}
\Sigma_{1}: \dot{x}_{1} & =A_{1} x_{1}+B_{1} \operatorname{sat}\left(u_{1}\right), \quad x_{1}(0)=0 \\
\Sigma_{2}: \dot{x}_{2} & =A_{2} x_{2}+B_{2} \operatorname{sat}\left(u_{2}\right), \quad x_{2}(0)=0 \\
y & =y_{V}+y_{P}=C_{1} x_{1}+C_{2} x_{2}
\end{aligned}\right.
$$

where the state $x_{1}=\left[\begin{array}{ll}y_{V} & \dot{y}_{V}\end{array}\right]^{T}, x_{2}=\left[\begin{array}{ll}y_{P} & \dot{y}_{P}\end{array}\right]^{T}$,

$$
\begin{array}{ll}
A_{1}=\left[\begin{array}{ll}
0 & 1 \\
0 & 0
\end{array}\right], \quad B_{1}=\left[\begin{array}{c}
0 \\
b_{1}
\end{array}\right], \quad C_{1}=\left[\begin{array}{ll}
1 & 0
\end{array}\right] \\
A_{2}=\left[\begin{array}{cc}
0 & 1 \\
a_{1} & a_{2}
\end{array}\right], & B_{2}=\left[\begin{array}{c}
0 \\
b_{2}
\end{array}\right], \quad C_{2}=\left[\begin{array}{ll}
1 & 0
\end{array}\right]
\end{array}
$$

and the saturation function $\operatorname{sat}\left(u_{i}\right)(i=1,2)$ is defined as

$$
\operatorname{sat}\left(u_{i}\right)=\operatorname{sgn}\left(u_{i}\right) \min \left\{\bar{u}_{i},\left|u_{i}\right|\right\}
$$

where $\bar{u}_{i}$ is the saturation level of the $i$ th control input.

The DSA model parameters in (3)-(5) are identified to match the measured responses with resonance compensator, which are given by

$$
\begin{array}{llrl}
b_{1} & =1.7 \times 10^{8}, & a_{1}=-10^{9}, & a_{2}=-3.1 \times 10^{4}(6) \\
b_{2}=4.3 \times 10^{8}, & \bar{u}_{1}=3 \mathrm{~V}, & \bar{u}_{2}=1.25 \mathrm{~V} .
\end{array}
$$

Fig. 2 shows that the identified models (dotted lines) can match the measured ones (solid lines) precisely in the frequency range of interest. Thus, from now on, we will take the DSA system in (2) as the plant model for further control design.

\section{B. State Estimator Design}

Since the head position $y$ is the only measurable signal for feedback control, we need to estimate the VCM and PZT actuator states by using the identified DSA model in (2)-(7). The state estimator is given by

$$
\hat{\dot{x}}=A_{D} \hat{x}+B_{D} \operatorname{sat}(u)+L\left(y-C_{D} \hat{x}\right)
$$

where $\hat{x}=\left[\begin{array}{llll}\hat{y}_{V} & \hat{\dot{y}}_{V} & \hat{y}_{P} & \hat{\dot{y}}_{P}\end{array}\right]^{T}, u=\left[\begin{array}{ll}u_{1} & u_{2}\end{array}\right]^{T}$,

$$
A_{D}=\left[\begin{array}{cc}
A_{1} & 0 \\
0 & A_{2}
\end{array}\right], \quad B_{D}=\left[\begin{array}{cc}
B_{1} & 0 \\
0 & B_{2}
\end{array}\right], \quad C_{D}=\left[\begin{array}{l}
C_{1} \\
C_{2}
\end{array}\right]^{T} .
$$

The state estimator gain $L$ can be calculated using the pole placement method [12] by selecting the well-damped estimator poles as two to six times faster than the DSA servo bandwidth.

Remark 1: According to the separation principle [12], the complete DSA control system in Fig. 3 is stable if the control laws $u_{1}$ and $u_{2}$ that are obtained assuming actual state feedback and the state estimator are both stable. Thus, we can design the control laws and the estimator separately yet used together. For the simplicity of design, we will present the VCM and PZT actuator control law $u_{1}$ and $u_{2}$ in Sections II-C and II-D assuming that the true state $x$ is available for feedback. However, the estimated states $\hat{x}$ will be used in final controller implementation.

\section{Vcm Actuator Control Design}

The role of the VCM actuator is to provide large seeking length beyond the PZT stroke limit. Thus, time-optimal control is critical to move the head position quickly from one track to another. The proximate time-optimal servomechanism (PTOS) is a practical near time-optimal controller that can accommodate plant uncertainty and measurement noise. Hence, we apply the PTOS control law [13] to the VCM actuator $\Sigma_{1}$ in (2) and the controller is independent of the PZT control loop. The PTOS control law is given by

$$
\begin{aligned}
u_{1} & =\operatorname{sat}\left[k_{2}\left(f\left(e_{1}\right)-\dot{y}_{V}\right)\right] \\
f\left(e_{1}\right) & = \begin{cases}\frac{k_{1}}{k_{2}} e_{1}, & \text { for }\left|e_{1}\right| \leq y_{l} \\
\operatorname{sgn}\left(e_{1}\right)\left(\sqrt{2 \bar{u}_{1} b_{1} \alpha\left|e_{1}\right|}-\frac{\bar{u}_{1}}{k_{2}}\right), & \text { for }\left|e_{1}\right|>y_{l}\end{cases} \\
e_{1} & =y_{r}-y_{V}
\end{aligned}
$$

where sat[·] is with the saturation level of $\bar{u}_{1}, \alpha$ is referred to as the acceleration discount factor, $k_{1}$ and $k_{2}$ are constant gains, and $y_{l}$ represents the size of a linear region. To make the functions $f\left(e_{1}\right)$ and $f^{\prime}\left(e_{1}\right)$ continuous such that the control input remains continuous as well, we have the following constraints:

$$
\begin{aligned}
\alpha & =\frac{2 k_{1}}{b_{1} k_{2}^{2}} \\
y_{l} & =\frac{\bar{u}_{1}}{k_{1}} .
\end{aligned}
$$

The PTOS control law introduces a linear region close to the setpoint to reduce the control chatter. In the region $\left|e_{1}\right| \leq y_{l}$, the control is linear, and thus, the gain $K=\left[\begin{array}{ll}k_{1} & k_{2}\end{array}\right]$ can be designed by any linear control techniques. For instance, using the pole placement method [12], we obtain a parameterized state feedback gain $K$ as

$$
K=\frac{1}{b_{1}}\left[4 \pi^{2} f_{1}^{2} \quad 4 \pi f_{1} \zeta_{1}\right]
$$


where $\zeta_{1}$ and $f_{1}$ (in hertz), respectively, represent the damping ratio and undamped natural frequency of the closed-loop system $C_{1}\left(s I-A_{1}+B_{1} K\right)^{-1} B_{1}$, whose poles are placed at $2 \pi f_{1}\left(-\zeta_{1} \pm j \sqrt{1-\zeta_{1}^{2}}\right)$.

In conventional DSA control systems, the VCM actuator controller is generally designed to have little overshoot such as by choosing a large damping ratio in (14). However, in our proposed control, a small damping ratio is chosen for a fast rise time and the resultant overshoot is within the PZT stroke limit, which can then be reduced by the PZT actuator under a composite nonlinear control law given in Section II-D.

\section{PZT Actuator Control Design}

The goal of the control design for the PZT actuator $\Sigma_{2}$ in (2) is to enable the PZT actuator to reduce the overshoot caused by the VCM actuator. We have the following step-by-step design procedure.

Step 1: Design a linear feedback control law

$$
u_{2 L}=F x_{2}
$$

where $F=\left[\begin{array}{ll}f_{1}^{\prime} & f_{2}^{\prime}\end{array}\right]$ is chosen such that the PZT actuator control system as given by

$$
\dot{x}_{2}=A_{2} x_{2}+B_{2} \operatorname{sat}\left(F x_{2}\right)
$$

is globally asymptotically stable (GAS) and the corresponding closed-loop system in the absence of input saturation $C_{2}\left(s I-A_{2}-B_{2} F\right)^{-1} B_{2}$ has a larger damping ratio and a higher undamped natural frequency than those of the VCM actuator control loop. To do this, we choose

$$
F=-B_{2}^{T} P
$$

where $P=P^{T}>0$ is the solution of the Lyapunov equation

$$
A_{2}^{T} P+P A_{2}=-Q
$$

for a given $Q=Q^{T}>0$. Note that the solution of $P$ exists since $A_{2}$ is Hurwitz. To involve the closed-loop properties explicitly with the control law, we define

$$
Q=\left[\begin{array}{cc}
q_{1} & 0 \\
0 & q_{2}
\end{array}\right], \quad q_{1}>0, \quad q_{2}>0
$$

where $q_{1}$ and $q_{2}$ are tuning parameters. Substituting (19) into (18) yields $P$, which gives the feedback gain (17) as follows:

$$
F=\frac{b_{2}}{2 a_{1} a_{2}}\left[a_{2} q_{1} \quad a_{1} q_{2}-q_{1}\right] .
$$

Moreover, the resulting poles of the closed-loop system $C_{2}(s I-$ $\left.A_{2}-B_{2} F\right)^{-1} B_{2}$ with (20) if complex conjugate have the undamped natural frequency and damping ratio as follows:

$$
\begin{aligned}
& f_{2}=\frac{1}{2 \pi} \sqrt{-\frac{b_{2}^{2}}{2 a_{1}} q_{1}-a_{1}} \\
& \zeta_{2}=\frac{b_{2}^{2} q_{1}-b_{2}^{2} a_{1} q_{2}-2 a_{1} a_{2}^{2}}{4 a_{1} a_{2} \sqrt{-\left(b_{2}^{2} / 2 a_{1}\right) q_{1}-a_{1}}} .
\end{aligned}
$$

Thus, we can easily achieve the desired $f_{2}$ and $\zeta_{2}$ by choosing a proper pair of $q_{1}$ and $q_{2}$.

Step 2: Construct the nonlinear feedback control law

$$
\begin{aligned}
u_{2 N} & =\gamma\left(y_{r}, y\right) H\left[\begin{array}{c}
y_{V}-y_{r} \\
\dot{y}_{V}
\end{array}\right] \\
H & =\frac{1}{b_{2}}\left[\begin{array}{ll}
\left(a_{1}+b_{2} f_{1}^{\prime}+b_{1} k_{1}\right) & \left(a_{2}+b_{2} f_{2}^{\prime}+b_{1} k_{2}\right)
\end{array}\right]
\end{aligned}
$$

where $H$ is taken to achieve desired closed-loop system dynamics, which will be clear in Section II-E; and $\gamma\left(y_{r}, y\right)$ is any nonnegative function locally Lipschitz in $y$, which is chosen to enable the PZT actuator to reduce the overshoot caused by the VCM actuator as the head position $y$ approaches the target track. The choice of $\gamma\left(y_{r}, y\right)$ will be discussed in Section II-E.

Step 3: Combine the linear and nonlinear feedback control laws derived in Steps 1 and 2 to form a composite nonlinear controller for the PZT actuator

$$
\begin{aligned}
u_{2} & =u_{1 L}+u_{2 N} \\
& =F x_{2}+\gamma\left(y_{r}, y\right) H\left[\begin{array}{c}
y_{V}-y_{r} \\
\dot{y}_{V}
\end{array}\right] .
\end{aligned}
$$

With the VCM actuator controller in (9) and the PZT actuator controller as given by (23), we have the following results regarding the step response of the DSA closed-loop system.

Lemma 1: Consider the DSA system in (2) with the VCM actuator $\Sigma_{1}$ under the PTOS control law (9) and the PZT actuator $\Sigma_{2}$ under the nonlinear control law (23) for any nonnegative function $\gamma\left(y_{r}, y\right)$ locally Lipschitz in $y$. Then, the composite control law will drive the head position $y$ to asymptotically track any step command input of amplitude $y_{r}$.

Proof: The VCM actuator closed-loop system under the PTOS control law can be represented as

$$
\dot{x}_{1}=A_{1} x_{1}+B_{1} \operatorname{sat}\left[k_{2}\left(f\left(e_{1}\right)-\dot{y}_{V}\right)\right]
$$

where $f\left(e_{1}\right)$ is defined in (10). It has been proved in [13] that the system (24) can track asymptotically any step command input of amplitude $y_{r}$, i.e.,

$$
\lim _{t \rightarrow \infty} y_{V}(t)=y_{r}, \quad \lim _{t \rightarrow \infty} \dot{y}_{V}(t)=0 .
$$

Next, we define a Lyapunov function $V=x_{2}^{T} P x_{2}$ with $P$ given in (18). Evaluating the derivative of $V$ along the trajectories of the system in (16) yields

$$
\begin{aligned}
\dot{V} & =\dot{x}_{2}^{T} P x_{2}+x_{2}^{T} P \dot{x}_{2} \\
& =x_{2}^{T}\left(A_{2}^{T} P+P A_{2}\right) x_{2}+2 B_{2}^{T} P x_{2} \operatorname{sat}\left(F x_{2}\right) \\
& =-x_{2}^{T} Q x_{2}-2 F x_{2} \operatorname{sat}\left(F x_{2}\right) \\
& \leq-x_{2}^{T} Q x_{2} \\
& <0 .
\end{aligned}
$$

Hence, the PZT actuator closed-loop system with linear feedback control only (16) is GAS. Furthermore, the PZT actuator closed-loop system with the composite nonlinear control law (23) can be expressed as

$$
\dot{x}_{2}=A_{2} x_{2}+B_{2} \operatorname{sat}\left(F x_{2}+u_{2 N}\right) .
$$


It is obvious that the system (27) satisfies the converging-input bounded-state (CIBS) property (see [15] for the definition) since $A_{2}$ is Hurwitz, $|\operatorname{sat}(\cdot)| \leq \bar{u}_{2}$, and the nonlinear control input $u_{2 N}$ has

$$
\lim _{t \rightarrow \infty} u_{2 N}(t)=0
$$

which can be easily deduced from (21) and (25).

The proof finishes by observing that the DSA closed-loop system formed by (24) and (27) has a cascaded structure and it satisfies the conditions of the theorem in [15]. It then follows that the cascade system formed by (24) and (27) is GAS at the origin. Thus, for the PZT actuator closed-loop system (27), we have

$$
\lim _{t \rightarrow \infty} x_{2}(t)=0
$$

and therefore

$$
\lim _{t \rightarrow \infty} y(t)=\lim _{t \rightarrow \infty}\left[C_{1} x_{1}(t)+C_{2} x_{2}(t)\right]=y_{r} .
$$

Remark 2: Lemma 1 shows that the value of $\gamma\left(y_{r}, y\right)$ does not affect the ability of the overall DSA closed-loop system to track asymptotically any step command input. However, from the perspective of transient performance, a proper choice of $\gamma\left(y_{r}, y\right)$ should be carried out to improve the performance of the overall DSA closed-loop system. This is the key property of the proposed control design.

\section{E. Selecting $\gamma\left(y_{r}, y\right)$ for Improved Performance}

The function $\gamma\left(y_{r}, y\right)$ is used to tune the control law to achieve our objective, a quick step response without a large overshoot. More specifically, we design the VCM actuator control loop to have a small damping ratio for a quick rise time and employ the PZT actuator control loop that is designed to be highly damped to reduce the overshoot caused by the VCM actuator as the head position $y$ approaches the target track. This control strategy implies that the dynamics of the DSA closed-loop system should be dominated by the VCM actuator control loop when the head position is far away from the target track, while dominated by the PZT actuator control loop when the head position approaches the target track. The purpose of the function $\gamma\left(y_{r}, y\right)$ is to fulfill a smooth transition from the VCM control loop to the PZT control loop.

Consider the DSA system (2) with the control laws in (9) and (23), and assume that the tracking error $\left(y_{r}-y\right)$ is small such that the control inputs do not exceed the limits and the control law (9) works within its linear region. Thus, the DSA closed-loop system can be expressed as

$$
\Sigma:\left\{\begin{array}{l}
\dot{x}=A x+B y_{r} \\
y=C x
\end{array}\right.
$$

where

$$
\begin{aligned}
A & =\left[\begin{array}{cc}
A_{1}-B_{1} K & 0 \\
\gamma\left(y_{r}, y\right) B_{2} H & A_{2}+B_{2} F
\end{array}\right] \\
B & =\left[\begin{array}{c}
B_{1} K \\
-\gamma\left(y_{r}, y\right) B_{2} H
\end{array}\right] \cdot\left[\begin{array}{l}
1 \\
0
\end{array}\right] \\
C & =\left[\begin{array}{ll}
C_{1} & C_{2}
\end{array}\right] .
\end{aligned}
$$

The DSA closed-loop transfer function from $y_{r}$ to $y$ can be obtained by

$$
\begin{aligned}
G(s) & =C(s I-A)^{-1} B \\
& =(1-\gamma) G_{1}(s)+\gamma G_{2}(s)
\end{aligned}
$$

where

$$
\begin{aligned}
G_{1}(s) & =\frac{b_{1} k_{1}}{s^{2}+b_{1} k_{2} s+b_{1} k_{1}} \\
G_{2}(s) & =\frac{-\left(a_{1}+b_{2} f_{1}^{\prime}\right)}{s^{2}-\left(a_{2}+b_{2} f_{2}^{\prime}\right) s-\left(a_{1}+b_{2} f_{1}^{\prime}\right)}
\end{aligned}
$$

are the closed-loop transfer function of the VCM and PZT actuator control loop, respectively.

At this point, it is clear that the DSA closed-loop system dynamics (32) change from the VCM control loop (33) to the PZT control loop (34) when $\gamma$ increases from 0 to 1 . This desired feature is due to the proper selection of $H$ in (22). From the perspective of zero placement, when $\gamma$ changes from 0 to 1 , the zeros of (32) is moved from the pole locations of the PZT control loop (33) to those of the VCM control loop (34). Since the zeros near the poles reduce the effects of the poles on the total response, we can use $\gamma$ to tune the system dynamics for desired performance. A similar control technique for singleinput single-output (SISO) linear systems can be found in [14], which, however, uses the nonlinear feedback law to increase the damping ratio of the closed-loop system poles to reduce the overshoot.

The function $\gamma\left(y_{r}, y\right)$ can be chosen as a function of the tracking error $y_{r}-y$. The following shows one choice of $\gamma$ :

$$
\gamma\left(y_{r}, y\right)=e^{-\beta\left|y_{r}-y\right|}
$$

where $\beta \geq 0$ is a tuning parameter. The function $\gamma\left(y_{r}, y\right)$ in (35) changes from 0 to 1 as $y \rightarrow y_{r}$. The parameter $\beta$ can be adjusted with respect to the amplitude of $y_{r}$ relative to the PZT actuator travel limit $\bar{y}_{2}$.

1) If $y_{r} \leq \bar{y}_{2}, \beta$ should be sufficiently small such that $\gamma$ converges to 1 quickly, which implies that the PZT control loop dominates the DSA closed-loop system dynamics over the whole control stage. In this case, the total settling time can be minimized because the PZT control loop has a much higher bandwidth than that of the VCM control loop.

2) If $y_{r}>\bar{y}_{2}, \beta$ should be large so as to divide the control stages into two parts. At the initial stage when the position output $y$ is far away from the final target track, $\gamma$ closes to 0 , which implies that the VCM control loop dominates the DSA closed-loop system dynamics to achieve a fast rise time while the PZT actuator is switched off because of its limited travel range. When the position output $y$ approaches the target track, $\gamma$ closes to 1 , which implies that the DSA closed-loop system dynamics is dominated by the PZT control loop that is highly damped. This high damping property can, in turn, imply that the PZT actuator is enabled to reduce the overshoot caused by the VCM actuator. 


\section{EXPERIMENTAL RESULTS AND DISCUSSION}

The proposed DSA tracking control has been applied to the dual-stage HDD in Fig. 1. The controller is implemented on a real-time DSP system (dSPACE 1103) with a sampling rate of $40 \mathrm{kHz}$. The state estimator gain is calculated as

$$
L=10^{4} \times\left[\begin{array}{llll}
5.7 & 51164 & 1.3 & 36952
\end{array}\right]^{T} .
$$

The PTOS controller in (9) for the VCM actuator is obtained by choosing $f_{1}=1200 \mathrm{~Hz}$ and thus $y_{l}=9 \mu \mathrm{m}$. The value of $\zeta_{1}$ is calculated as a function of $y_{r}$ such that the VCM has a fast rise time while the resultant overshoot approximately equals to the PZT stroke limit (i.e., $0.5 \mu \mathrm{m}$ ). Since the overall response is dominated by the PZT loop when $y_{r} \leq 0.5 \mu \mathrm{m}$, we can set $\zeta_{1}$ as any constant less than 1 for $y_{r} \leq 0.5 \mu \mathrm{m}$. When $y_{r}>0.5$ $\mu \mathrm{m}$, the solution of $\zeta_{1}$ can be numerically derived such that the overshoot approximately equals to $0.5 \mu \mathrm{m}$. For example, one choice of $\zeta_{1}$ is given by

$$
\zeta_{1}\left(y_{r}\right)= \begin{cases}0.3, & y_{r} \leq 0.5 \mu \mathrm{m} \\ \frac{1.3 \times\left(\ln \left(y_{r}\right)+0.7\right)}{\sqrt{\pi^{2}+\left(\ln \left(y_{r}\right)+0.7\right)^{2}}}, & y_{r}>0.5 \mu \mathrm{m} .\end{cases}
$$

Hence, the linear gain $K$ is given by

$$
K=\left[\begin{array}{ll}
0.33 & 0.00009 \zeta_{1}\left(y_{r}\right)
\end{array}\right] .
$$

Note that if the overshoot caused by the VCM is larger than the PZT stroke limit, asymptotical stability can still be guaranteed according to Lemma 1. However, an overshoot that exceeds the PZT span will exist in the overall step response, which accordingly results in no reduction of the overall settling time. Therefore, the proposed method is effective only when the VCM controller is designed to have an overshoot within the PZT span.

For the PZT control design, we choose $q_{1}=1.3$ and $q_{2}=$ $9 \times 10^{-9}$, and thus, the linear feedback gain $F$ is given by

$$
F=-\left[\begin{array}{ll}
0.28 & 0.00007
\end{array}\right]
$$

which results in $f_{2}=5300 \mathrm{~Hz}$ and $\zeta_{2}=0.95$. The nonlinear feedback gain is given by

$$
H=-\left[\begin{array}{ll}
2.4 & 0.00015-0.000035 \zeta_{1}\left(y_{r}\right)
\end{array}\right]
$$

with $\zeta_{1}\left(y_{r}\right)$ in (36). The nonlinear function (35) is chosen as

$$
\gamma\left(y_{r}, y\right)= \begin{cases}e^{-0.05\left|y-y_{r}\right|}, & y_{r} \leq 0.5 \mu \mathrm{m} \\ e^{-0.5\left|y-y_{r}\right|}, & y_{r}>0.5 \mu \mathrm{m} .\end{cases}
$$

In order to compare the proposed control with the conventional control where the VCM control loop is generally tuned to have no overshoot, we choose $\zeta_{1}=0.9$ for any $y_{r}$ and retain the other tuning parameters, then following the same design procedure yields a conventional controller that is used for comparison with our proposed controller. Moreover, we define the settling time to be the time that it takes for the head position $y$ to enter and remain within $\pm 5 \%$ of the target track.

\section{A. Track Seeking}

We first evaluate the track seeking responses within the PZT stroke limit. Fig. 4 shows the result for $y_{r}=0.5 \mu \mathrm{m}$. We can
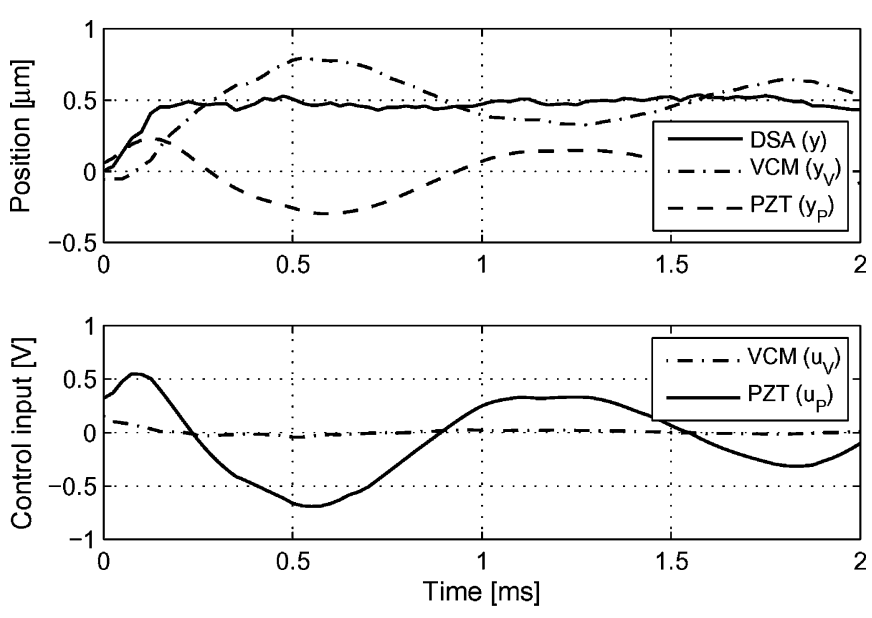

(a)
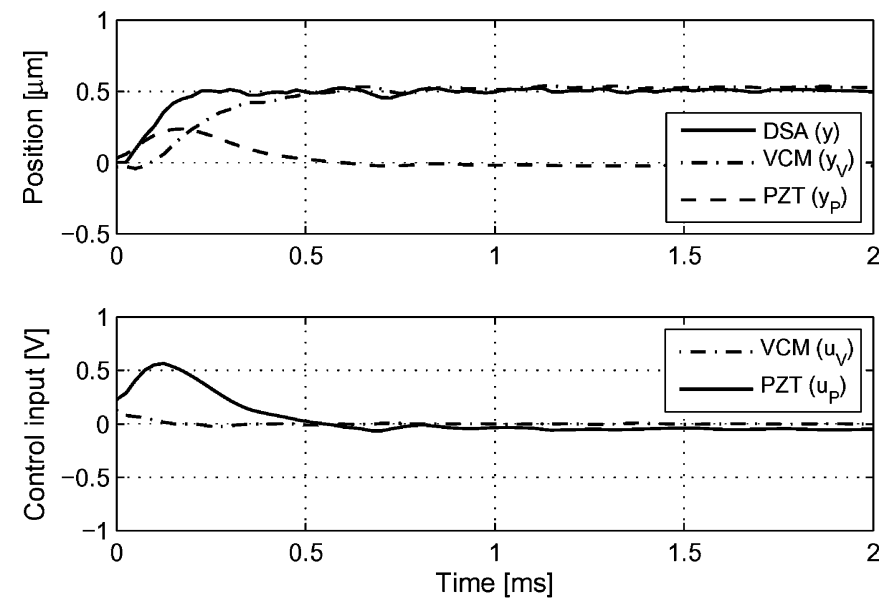

(b)

Fig. 4. Seeking responses to $y_{r}=0.5 \mu \mathrm{m}$. Within the PZT stroke limit, the settling time in both control are similarly $0.25 \mathrm{~ms}$. (a) Proposed control. (b) Conventional control.

TABLE I

COMPARISON OF THE SETTLING TIME IMPROVEMENT

\begin{tabular}{c|c|c|c}
\hline $\begin{array}{c}\text { Seeking length } \\
(\mu \mathrm{m})\end{array}$ & \multicolumn{2}{|c|}{ Settling Time (ms) } & $\begin{array}{c}\text { Improvement } \\
(\%)\end{array}$ \\
\cline { 2 - 3 } & Conventional & Proposed & 0 \\
\hline 0.5 & 0.25 & 0.25 & 21 \\
\hline 2 & 0.38 & 0.3 & 25 \\
\hline 3 & 0.4 & 0.3 & 14 \\
\hline 5 & 0.42 & 0.36 & 2.5 \\
\hline 7 & 0.41 & 0.4 & 0 \\
\hline 10 & 0.45 & 0.45 & \\
\hline
\end{tabular}

see that the settling times under the proposed control and the conventional control are almost the same. This is because within the PZT stroke limit, the PZT control loop dominates the DSA closed-loop system dynamics irrespective of the fact which VCM control loop is tuned. However, when the seeking length is beyond the PZT stroke limit as shown in Figs. 5 and 6, the settling time under the proposed control is significantly reduced compared with the conventional control. The tests of other seeking lengths are also conducted. All the results in terms of the settling time are summarized in Table I for easy comparison. 

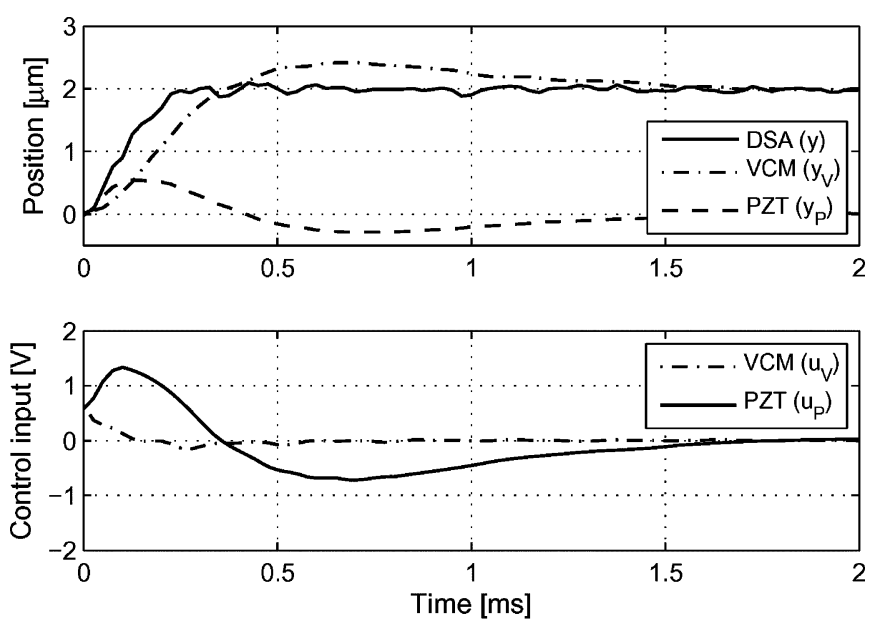

(a)
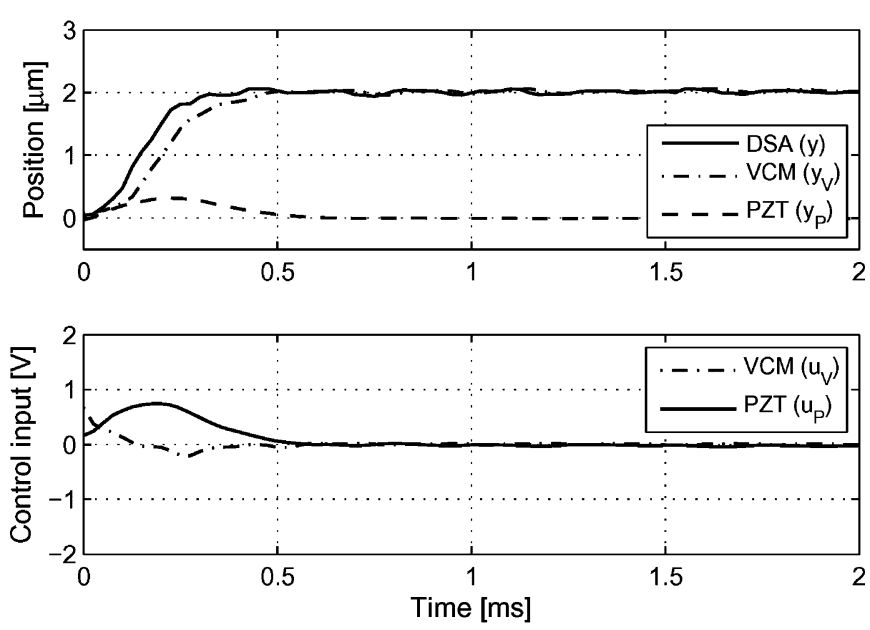

(b)

Fig. 5. Seeking responses to $y_{r}=2 \mu \mathrm{m}$. The settling time in the conventional control is $0.38 \mathrm{~ms}$, which is reduced to $0.3 \mathrm{~ms}$ in the proposed control. (a) Proposed control. (b) Conventional control.

It is shown that the proposed control can further reduce the settling time by more than $14 \%$ for short-span seeking between 2 and $5 \mu \mathrm{m}$. However, the reduction ratio decreases for longspan seeking because the PZT stroke limit is slight relative to large seeking lengths.

\section{B. Track Following}

Finally, we evaluate the position error signal (PES) of the proposed DSA control system in Fig. 3 by injecting the disturbance $d$ and setting $y_{r}=0$. The disturbance signals are collected from a real HDD, which consist of repeatable runout (RRO) and nonrepeatable runout (NRRO). The experimental steady-state PES (i.e., PES $=y-d$ ) is shown in Fig. 7. It is obvious that the dual-stage servo significantly decreases the PES amplitude compared with the VCM only single-stage servo. The PES $3 \sigma$ value is reduced from 167.1 to $143.4 \mathrm{~nm}$, which is a $14 \%$ reduction ratio. Moreover, Fig. 8 shows the power spectrum density (PSD) of the PES, which indicates that the dual-stage servo yields a considerable improvement in the frequency range from
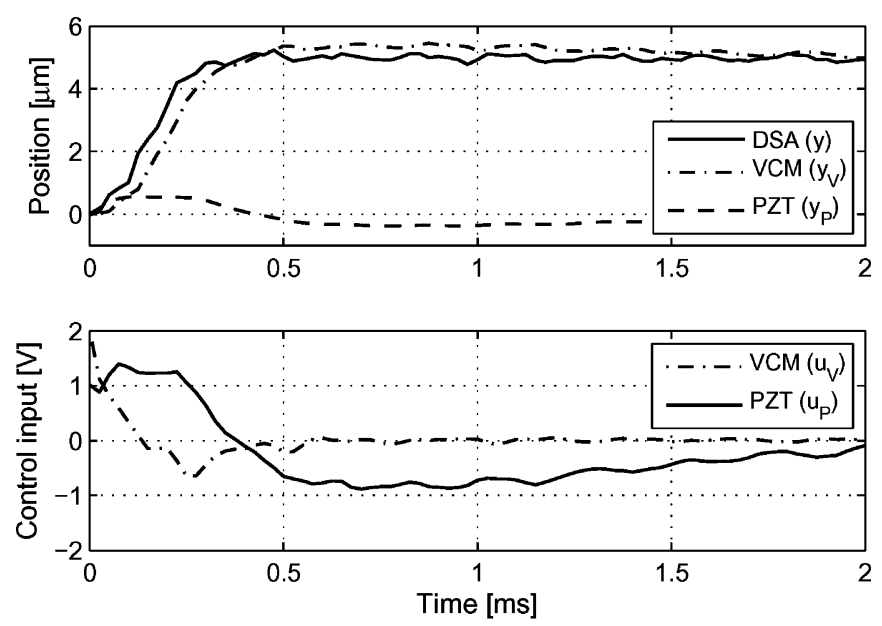

(a)
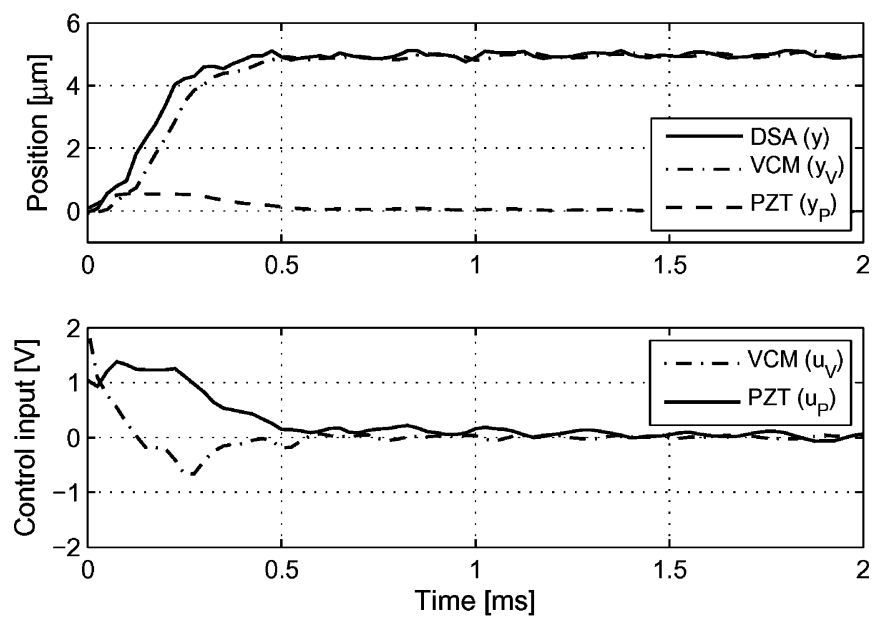

(b)

Fig. 6. Seeking responses to $y_{r}=5 \mu \mathrm{m}$. The settling time in the conventional control is $0.42 \mathrm{~ms}$, which is reduced to $0.36 \mathrm{~ms}$ in the proposed control. (a) Proposed control. (b) Conventional control.
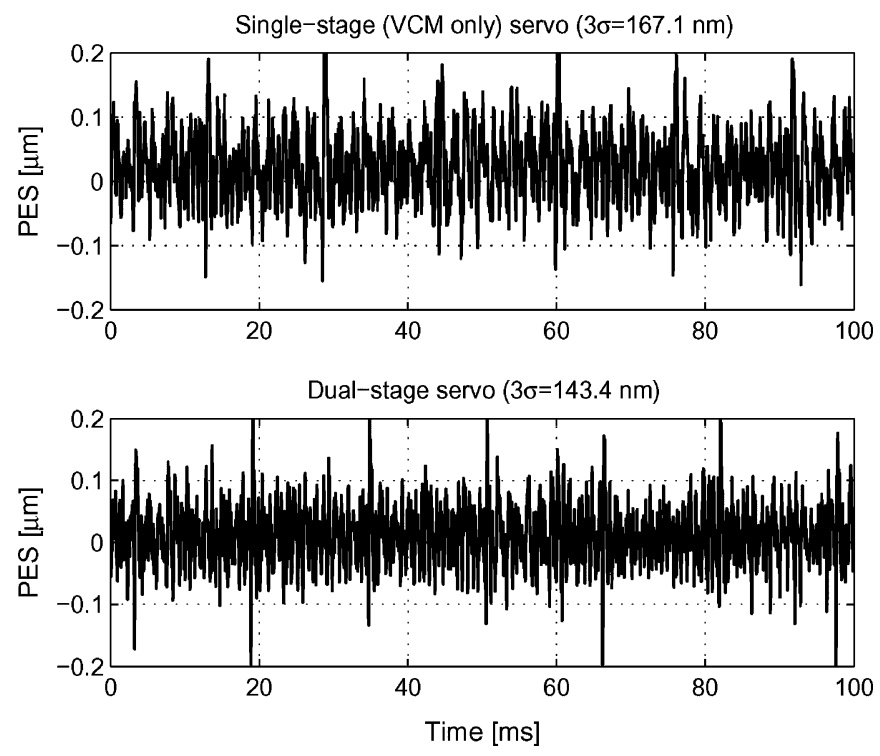

Fig. 7. Position error signals. 

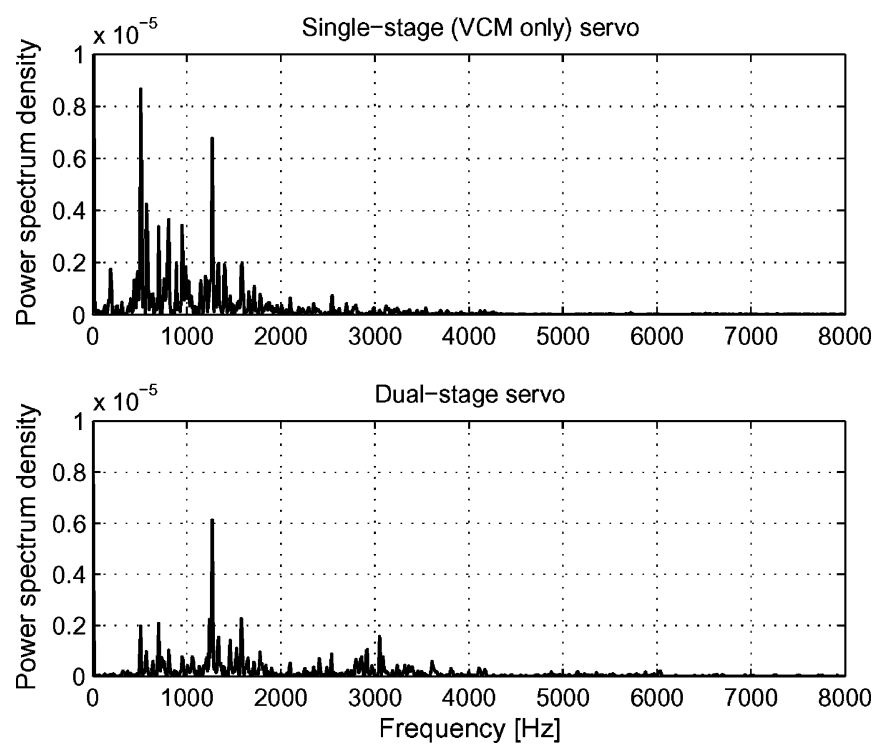

Fig. 8. PSD of the PES.

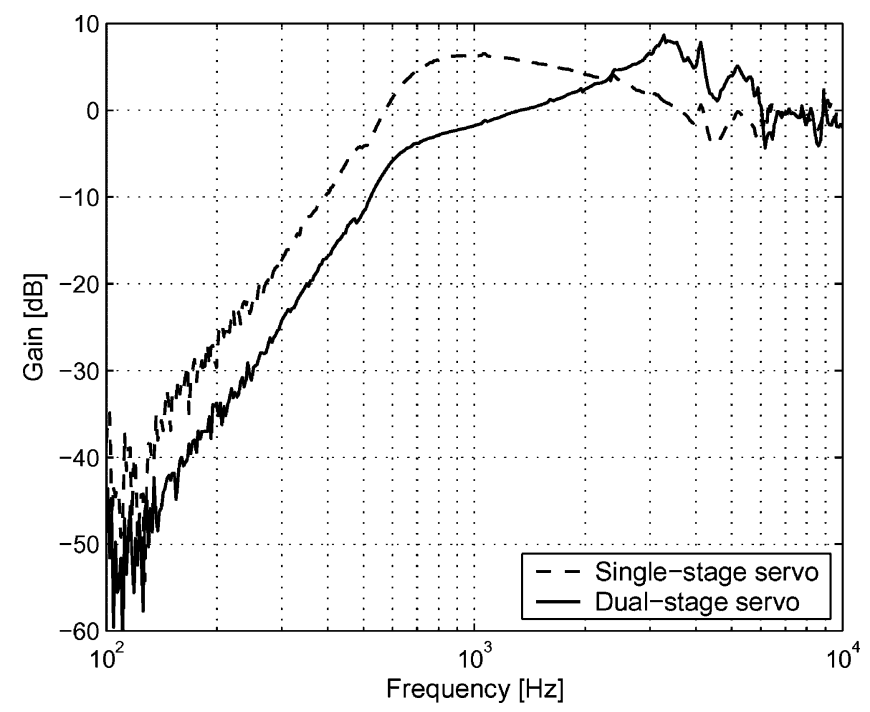

Fig. 9. Measured frequency responses of sensitivity function.

0 to $2500 \mathrm{~Hz}$. Above $2500 \mathrm{~Hz}$, the dual-stage servo leads to a bit small degradation. This tradeoff is due to the waterbed effect in linear control systems, which can be more easily observed from the measured frequency responses of the sensitivity function as shown in Fig. 9.

\section{CONCLUSION}

In this paper, we have proposed a nonlinear tracking control method for a disk drive DSA system with a PZT microactuator. Distinct from the conventional control method, the VCM actuator control loop is designed to have a small damping ratio for a fast rise time. The PZT actuator control loop is then enabled by a nonlinear control law to reduce the overshoot caused by the VCM actuator as the system output approaches the target track.
Experimental results showed that the proposed control can significantly reduce the settling time in short-span seeking by more than $14 \%$ compared with the conventional control. Moreover, the proposed DSA servo system can achieve a $14 \%$ reduction ratio of the PES $3 \sigma$ compared with the VCM only servo system.

\section{REFERENCES}

[1] K. Mori, T. Munemoto, H. Otsuki, Y. Yamaguchi, and K. Akagi, “Adualstage magnetic disk drive actuator using a piezoelectric device for a high track density," IEEE Trans. Magn., vol. 27, no. 6, pp. 5298-5300, Nov. 1991.

[2] T. Imamura, M. Katayama, Y. Ikegawa, T. Ohwe, R. Koishi, and T. Koshikawa, "MEMS-based integrated head/actuator/slider for hard disk drives," IEEE/ASME Trans. Mechatron., vol. 3, no. 3, pp. 166-174, Sep. 1998.

[3] R. Evans, J. Griesbach, and W. Messner, "Piezoelectric microactuator for dual stage control," IEEE Trans. Magn., vol. 35, no. 2, pp. 977-982, Mar. 1999.

[4] S. Schroeck, W. Messner, and R. McNab, "On compensator design for linear time-invariant dual-input single-output systems," IEEE/ASME Trans. Mechatron., vol. 6, no. 1, pp. 50-57, Mar. 2001.

[5] X. Huang and R. Horowitz, "Robust controller design of a dual-stage disk drive servo system with an instrumented suspension," IEEE Trans. Magn., vol. 41, no. 8, pp. 2406-2413, Aug. 2005.

[6] H. Numasato and M. Tomizuka, "Settling control and performance of a dual-actuator system for hard disk drives," IEEE/ASME Trans. Mechatron, vol. 8, no. 4, pp. 431-438, Dec. 2003.

[7] G. Herrmann, M. Turner, I. Postlethwaite, and G. Guo, "Practical implementation of a novel anti-windup scheme in a HDD-dual-stage servo system," IEEE/ASME Trans. Mechatron, vol. 9, no. 3, pp. 580-592, Sep. 2004.

[8] T. Shen and M. Fu, "High precision and feedback control design for dual-actuator systems," in Proc. IEEE Conf. Control Appl., Toronto, ON, Canada, Aug. 2005, pp. 956-961.

[9] M. Kobayashi and R. Horowitz, "Track seek control for hard disk dualstage servo systems," IEEE Trans. Magn., vol. 37, no. 2, pp. 949-954, Mar. 2001.

[10] S. Lee and Y. Kim, "Minimum destructive interference design of dualstage control systems for hard disk drives," IEEE Trans. Control Syst. Technol., vol. 12, no. 4, pp. 517-531, Jul. 2004.

[11] B. Hredzak, G. Herrmann, and G. Guo, "A proximate-time-optimal control design and its application to a hard disk drive dual-stage actuator system," IEEE Trans. Magn., vol. 42, no. 6, pp. 1708-1715, Jun. 2006.

[12] G. F. Franklin, J. D. Powell, and A. Emami-Naeini, Feedback Control of Dynamic Systems, 3rd ed. Reading, MA: Addison-Wesley, 1994.

[13] M. Workman, Adaptive Proximate Time-Optimal Servomechanisms, Ph.D. dissertation, Stanford Univ.. Stanford, CA, 1987.

[14] Z. Lin, M. Pachter, and S. Banda, "Toward improvement of tracking performance-nonlinear feedback for linear systems," Int. J. Control, vol. 70, pp. 1-11, 1998.

[15] E. Sontag, "Remarks on stabilization and input-to-state stability," in Proc. IEEE Conf. Decis. Control, Tampa, FL, Dec.1989, vol. 2, pp. 1376-1378.

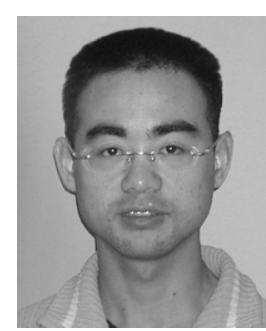

Jinchuan Zheng received the B.Eng. and M.Eng. degrees in mechatronics engineering from Shanghai Jiao Tong University, Shanghai, China, in 1999 and 2002 , respectively, and the Ph.D. degree in electrical engineering from Nanyang Technological University, Singapore, in 2006.

In 2005, he joined the Australian Research Council (ARC) Centre of Excellence for Complex Dynamic Systems and Control, School of Electrical and Computer Engineering, The University of Newcastle, Callaghan, NSW, Australia, as a Research Academic. His current research interests include nanopositioning system design, vibration analysis, sensing and control, and advanced control applications to mechatronics. 


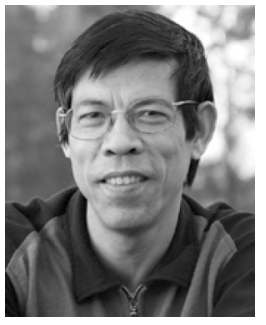

Minyue Fu (S'84-M'87-SM'94-F'02) received the Bachelor's degree in electrical engineering from the University of Science and Technology of China, Hefei, China, in 1982, and the M.S. and Ph.D. degrees in electrical engineering from the University of Wisconsin, Madison, in 1983 and 1987, respectively.

From 1987 to 1989, he was as an Assistant Professor in the Department of Electrical and Computer Engineering, Wayne State University, Detroit, MI. In 1989, he joined the Department of Electrical and Computer Engineering, The University of Newcastle, Callaghan, NSW, Australia, where he has been the Head of the Department and the Head of School, and is currently the Chair Professor in Electrical Engineering. During 1995 and 1996, he was a Visiting Associate Professor at the University of Iowa, Iowa City, and during 2002, a Senior Fellow/Visiting Professor at Nanyang Technological University, Singapore. His current research interests include control systems, signal processing, and communications.

Dr. Fu was an Associate Editor for the IEEE TRANSACTIONS ON AUTOMATIC CONTROL. He was an Associate Editor for Automatica and the Journal of Optimization and Engineering.

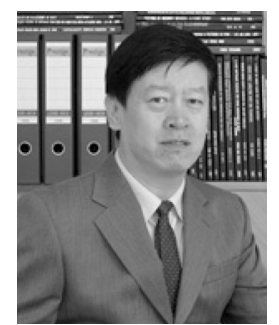

Youyi Wang (S'90-M'91-SM'96) received the B.Eng. degree from the University of Science and Technology, Beijing, China, in 1982, the M.Eng. degree from Tsinghua University, Beijing, in 1984, and the Ph.D. degree from The University of Newcastle, Callaghan, NSW, Australia, in 1991, all in electrical engineering.

In 1991, he joined Nanyang Technological University, Singapore, where he is currently an Associate Professor in the School of Electrical and Electronic Engineering. He is currently the Deputy Director of the Center for Smart Energy Systems and the Protective Technology Research Center, and the Principal Investigator of the Center for Mechanics of MicroSystems. He is the author or coauthor of over 240 published referred international journals and conference papers on system theory, control theory, stability analysis, information storage system, and control theory application. His current research interests include the areas of nonlinear control, robust control, control and applications of control theory to power systems, electric drive systems, information storage systems, fuel cell systems, renewable energy systems, and microelectromechanical systems (MEMS).

Dr. Wang is a Senior Member of the IEEE Control Systems Society and the IEEE Power Engineering Society, and a Treasurer of the IEEE Singapore Control Systems Chapter.

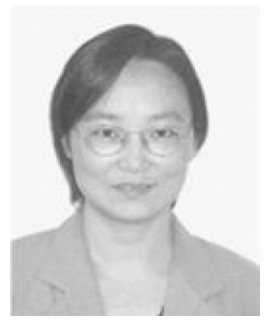

Chunling Du (M'00-SM'07) received the B.E. and M.E. degrees in electrical engineering from Nanjing University of Science and Technology, Nanjing, China, in 1992 and 1995, respectively, and the Ph.D. degree in electrical engineering from Nanyang Technological University, Singapore, in 2000.

In 1999, she joined the Agency for Science, Technology and Research $(A *$ STAR) Data Storage Institute (DSI), Singapore, as a Research Engineer, where she is currently a Senior Research Engineer in the Mechatronics and Recording Channel Division. She is also an Adjunct Assistant Professor at Nanyang Technological University. She is the author or coauthor of several published papers and also coauthored the monograph H-infinity Control and Filtering of Two-dimensional Systems (Springer-Verlag, 2002). Her current research interests include optimal and robust control, vibration modeling and control, advanced control and mechatronics for nanopositioning systems, signal processing, and multidimensional systems. 